УДК 519.873

\title{
Synthesis of the Radio-navigation Signals Parameters Measured by an Exploded Antenna Elements
}

\author{
Valery N. Tyapkin* \\ Dmitry D. Dmitriev ${ }^{\dagger}$ \\ Nikolay S. Kremez $z^{\ddagger}$ \\ Igor V. Tyapkin ${ }^{\S}$ \\ Siberian Federal University \\ Svobodny, 79, Krasnoyarsk, 660041 \\ Russia
}

Received 10.9.2019, received in revised form 18.10.2019, accepted 25.11.2019

Modern GNSS navigation equipment has a significant drawback - low noise immunity. To solve this problem, they are based on phased array antennas using adaptive signal processing algorithms. The final effect of such processing is determined by the level and completeness of using the existing differences, as well as the quality of accounting for each of them, depending on the degree of fame of the statistical characteristics of signals and interference. The article discusses the theory of synthesis of an algorithm for measuring the parameters of radio navigation signals received on spaced antennas under the influence of interference.

Keywords: phased array, adaptive algorithms, normal distribution law, vector of estimated parameters, vector of useful signals.

DOI: $10.17516 / 1997-1397-2019-12-6-772-779$

\section{Introduction}

For efficient functioning in real conditions of parametric a priori uncertainty and a dynamic change in the statistical characteristics of interference, the parameters of adaptive systems must be promptly changed in accordance with the interference environment. The most substantiated theoretically and tested in practice are the methods of the Markov theory of optimal filtration. This theory has been fully and strictly developed in a number of works [1-12].

The problem of optimal filtering of the measured navigation parameters in general is formulated as follows:

a) it is required to obtain an optimal estimate of the state vector of the navigation parameters $\lambda(t)$. A vector formation model is described by a stochastic differential equation [3]:

$$
d \lambda / d t=\mathbf{F}(t) \boldsymbol{\lambda}(t)+\mathbf{G}(t) \boldsymbol{\xi}(t), \quad \lambda(0)=\lambda_{0} .
$$

\footnotetext{
*tyapkin58@mail.ru

†dmitriev121074@mail.ru

${ }_{\ddagger}$ nkremez@sfu-kras.ru

$\S$ aronakss@yandex.ru

(c) Siberian Federal University. All rights reserved
} 
where $\mathbf{F}(t), \mathbf{G}(t)$ are functions of time; $\boldsymbol{\xi}(t)$ is white Gaussian noise with two-sided spectral density $\mathbf{s}_{\xi} ; \lambda_{0}$ is a random number distributed according to the Gaussian law with zero expectation and dispersion $D_{\lambda 0}$.

Under the assumption of noise independence, the posterior probability density of the state vector satisfies the Stratonovich equation and has a Gaussian approximation.

b) The observed process, i.e., an additive mixture of received signals and interference, is represented as

$$
\mathbf{Y}(t)=\mathbf{S}_{C}(t, \lambda(t))+\mathbf{n}(t), t \geqslant t_{0},
$$

where $\mathbf{S}_{C}(t, \boldsymbol{\lambda}(t))$ is vector of useful signals; $\boldsymbol{\lambda}(t)$ is vector of estimated parameters; $\mathbf{n}(t)$ is white Gaussian noise vector with zero expectation and correlation function.

In most cases, the normal law of distribution of received signals is justified, since in the narrow-band frequency receiving paths the interference is normalized.

c) Ensuring the maximum pradimilarity ratio using the quadratic loss function and the Gaussian approximation of the posterior probability density (which is true if the estimation errors do not go beyond the linear portion of the characteristics of the optimal discriminators).

Thus, the problem of measuring radio navigation parameters in a general form can be formulated as follows:

Using observations (2) and a priori information on the statistical characteristics of processes $\boldsymbol{\lambda}$ and $\mathbf{n}(t)$, it is necessary to form an estimate $\boldsymbol{\lambda}$ that is best in one sense or another.

\section{Initial conditions and ratios for synthesis}

Consider a flat antenna array with m omnidirectional antenna elements $A_{j}, j=\overline{1, m}$ (Fig. 1).

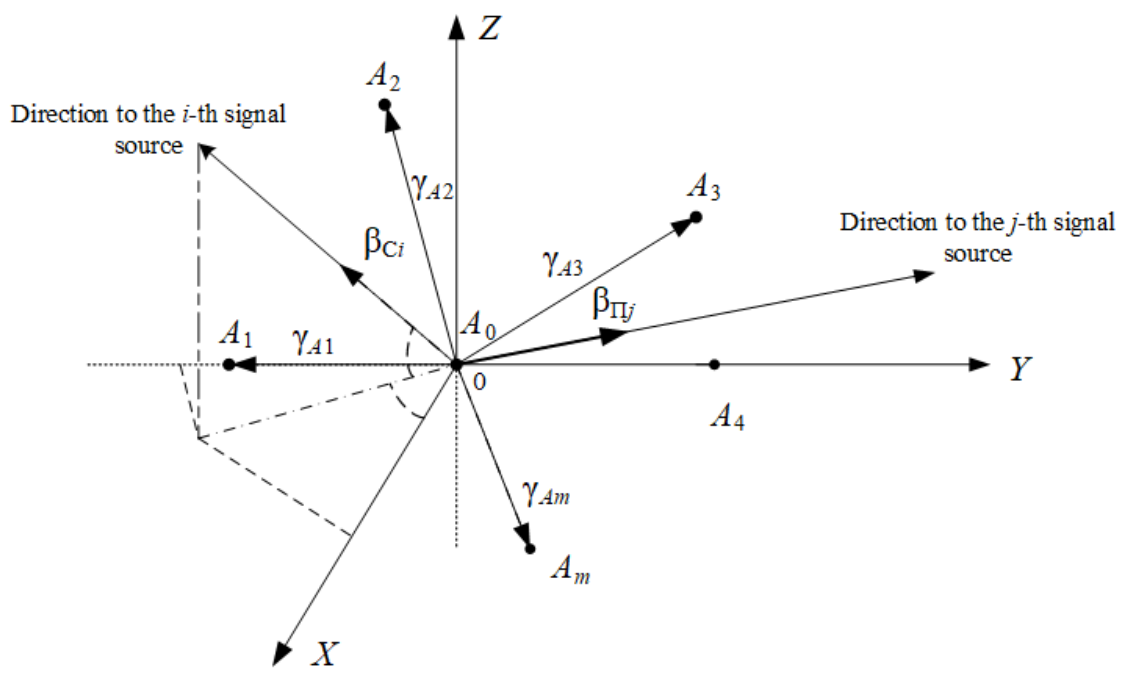

Fig. 1. Geometric interpretation of the location of the elements of the antenna array and received signals

We introduce the $O X Y Z$ coordinate system, the origin of which is aligned with the zero antenna element, and the $O X Y$ plane coincides with the plane of the antenna array. The position 
of each $i$-th antenna element in the $O X Y Z$ coordinate system is characterized by a vector $\gamma_{A i}$. Let a flat wavefront from signals and interference come to the antenna array. Directions to the radiation sources of signals are set by unit vectors $\beta_{C i}$, and the direction to sources of interference are set by unit vectors $\beta_{\Pi j}$.

At the output of the $l$-th antenna element we have a continuous signal [2]:

$$
y_{l}(t)=\sum_{i=1}^{n} \sqrt{P_{c i}} s_{c_{i}, l}\left(t, \lambda_{i, l}\right)+\sum_{j=1}^{p} \sqrt{P_{\Pi, j}} s_{\Pi, j, l}(t)+n_{l}(t), l=\overline{1, m},
$$

where $s_{c_{i}, l}\left(t, \lambda_{i, l}\right)$ is signal of unit power of the $i$-th navigation satellite on the $l$-th antenna element; $P_{c i}$ is signal strength of the $i$-th navigation satellite; $P_{п i}$ is power of the $j$-th interference signal; $n_{l}(t)$ is white Gaussian noise with correlation matrix $M\left[n_{l}(t) n_{l}(t+\tau)\right]=$ $=\frac{N_{0}}{2} \delta(\tau), M\left[n_{m}(t) n_{l}\left(t_{1}\right)\right]=0, m \neq l$, for any $t$ and $t_{1} ; \lambda_{i, l}$ is vector of parameters (generally changing over time) to be evaluated.

When synthesizing and analyzing meters of radio navigation parameters of signals received at spaced antennas, it is necessary to specify a signal-noise situation [1-3], which includes statistical models:

- useful signals $\dot{S}_{c_{i}, l}\left(t_{k}, \lambda_{i, l}\right), l=\overline{1, m}, i=\overline{1, n}$

- interference signals $\dot{S}_{\Pi_{j, l}}\left(t_{k}\right), l=\overline{1, m}, j=\overline{1, p}$

- vectors of estimated parameters $\lambda_{i, l ; k}, l=\overline{1, m}, i=\overline{1, n}$

The methods for setting the signal-noise environment will affect the implementation of algorithms for optimal (quasi-optimal) processing and obtaining estimates of the vector of informative parameters.

GNSS uses narrowband signals [1-3], the spectrum width of which is substantially less than the carrier frequency. It is convenient to describe such signals with complex amplitudes determined with respect to a fixed carrier frequency $\omega_{0}=2 \pi f_{0}$.

In modern GNSS signal receivers, digital processing is performed. We record the observations in discrete time [2].

$$
\dot{y}_{l}\left(t_{k}\right)=\sum_{i=1}^{n} \sqrt{P_{\mathrm{ci}}} \dot{s}_{c_{i}, l}\left(t_{k}, \lambda_{i, l ; k}\right)+\sum_{j=1}^{p} \sqrt{P_{\Pi_{j}}} \dot{s}_{\Pi_{j}, l}\left(t_{k}\right)+\dot{n}_{l}\left(t_{k}\right), l=\overline{1, m},
$$

where

$$
\begin{aligned}
& \dot{S}_{c_{i}, l}\left(t_{k}, \lambda_{i, l}\right)=H_{i, l}\left(\alpha_{i, l ; k}\right) \dot{\tilde{S}}_{c_{i}, l}\left(t_{k}, \lambda_{i, l ; k}\right), \\
& \dot{S}_{\Pi_{j}, l}\left(t_{k}\right) \equiv \dot{s}_{\Pi_{j}, l}\left(t_{k}, \alpha_{\Pi_{j}, l ; k}\right)=\dot{C}_{j, l}\left(\alpha_{\Pi_{j}, l ; k}\right) \dot{\tilde{s}}_{\Pi_{j}, l}\left(t_{k}\right), \\
& \dot{\tilde{S}}_{c_{i}, l}\left(t_{k}, \lambda_{i, l ; k}\right)=h_{R C}\left(t_{k}-\tau_{i, l ; k}\right) h_{N M}\left(t_{k}-\tau_{i, l ; k}\right) e^{j\left(\varphi_{i, k}\right)} \text { - a factor describing the temporal }
\end{aligned}
$$
structure of the received signal;

$\varphi_{k, i}=\varphi_{k}+\omega_{d, k} i T_{d}$ - phase of the high-frequency signal, the same for each antenna element;

$\mathbf{H}_{i}=\left|1 e^{j\left(\psi_{i, 2}\right)} \cdots e^{j\left(\psi_{i, l}\right)} \cdots e^{j\left(\psi_{i, m}\right)}\right|^{T}-$ the vector of the phase distribution of the signal of the $i$-th navigation satellite on the antenna elements;

$\psi_{i, l}=\frac{2 \pi \boldsymbol{\rho}_{A_{l}} \beta_{s, i}}{\lambda}-$ the phase change of the radio signal of the $i$-th navigation satellite on the $l$-th antenna element relative to the first;

$\mathbf{C}_{j}=\left|1 e^{j\left(\psi_{\mathrm{n}_{J}, 2}\right)} \cdots e^{j\left(\psi_{\mathrm{n} J, l}\right)} \cdots e^{j\left(\psi_{\mathrm{n}_{J}, m}\right)}\right|^{T}$ - vectors of phase distributions of the $j$-th interference signal on the antenna elements; 
$\psi_{\Pi ; j, l}=\frac{2 \pi \boldsymbol{\rho}_{A_{l}} \boldsymbol{\beta}_{\Pi, j}}{\lambda}-$ the phase difference of the interfering signal on the $l$-th antenna element relative to the first;

$\dot{n}_{l, k}$ - complex white Gaussian noise for which $M\left[\dot{n}_{l, k} \dot{n}_{l, m}^{*}\right]=2 \sigma_{n}^{2} \delta_{k, m}, \sigma_{n}^{2}=N_{0} /\left(2 T_{d}\right)$, $M\left[\dot{n}_{m, k} \dot{n}_{l, v}^{*}\right]=0, m \neq l ; \lambda_{i, l ; l}$ - vector of parameters to be evaluated. Combine observations (4) into a vector observation:

$\mathbf{Y}\left(t_{k}\right)=\left|\dot{y}_{1}\left(t_{k}\right) y_{2}\left(t_{k}\right) \ldots \dot{y}_{m}\left(t_{k}\right)\right|^{\mathrm{T}}$

The set of samples $\mathbf{Y}\left(t_{k}\right), l=\overline{1, m}, k=1,2, \ldots$ will be called spatio-temporal observations.

$$
\mathbf{Y}\left(t_{k}\right)=\mathbf{H}_{k} \mathbf{S}_{c}\left(t_{k}, \boldsymbol{\lambda}_{k}\right)+\mathbf{C}_{k} \mathbf{S}_{\Pi}\left(t_{k}\right)+\mathbf{n}_{\left(t_{k}\right)}
$$

where

$\mathbf{H}_{k}=\left(\mathbf{H}_{1, k} \mathbf{H}_{2, k} \ldots \mathbf{H}_{i, k} \ldots \mathbf{H}_{N, k}\right)$,

$\mathbf{C}_{k}=\left(\mathbf{C}_{1, k} \mathbf{C}_{2, k} \ldots \mathbf{C}_{j, k} \ldots \mathbf{C}_{P, k}\right)$,

$\mathbf{H}_{k}$ and $\mathbf{C}_{k}$ are the vectors of the phase distributions of the signal and interference on the antenna elements;

$\tilde{\mathbf{n}}_{t}$ - white Gaussian noise modeling interference sources;

$\mathbf{n}_{0 t}$ - vector of independent white Gaussian noises (internal noise in the channels).

If the total noise designate

$$
\mathbf{n}_{k}=\mathbf{C}_{k} \mathbf{S}_{\Pi k}+\mathbf{n}_{0 k}
$$

observation equation (5) takes the form

$$
\mathbf{Y}_{k}=\mathbf{H}_{k} \mathbf{S}_{c k}\left(\boldsymbol{\lambda}_{k}\right)+\mathbf{n}_{k}
$$

Synthesis optimal adaptive algorithm navigation measurement parameters (angles of orientation of the object) from the antenna array on the signals in a GNSS unknown interference situation requires the evaluation of joint probability density of posteriori $p(\boldsymbol{\lambda}, \boldsymbol{\Phi} \mid \dot{\mathbf{Y}})$, where $\boldsymbol{\lambda}$ is the vector of the required informative parameters, $\boldsymbol{\Phi}$ - interference correlation matrix, $\mathbf{Y}-$ vector of observed parameters at the output of the antenna array. In the general case, an estimate of the required informative parameters $\boldsymbol{\lambda}$ can be obtained from the relation:

$$
p(\boldsymbol{\lambda} / \mathbf{Y})=\int p(\boldsymbol{\lambda}, \boldsymbol{\Phi} / \mathbf{Y}) p(\mathbf{\Phi} / \mathbf{Y}) d \mathbf{\Phi} .
$$

The correlation matrix of interference $\boldsymbol{\Phi}$ can be calculated with high accuracy, therefore, the expression can (8) be represented as:

$$
\left\{\begin{array}{l}
\hat{\boldsymbol{\Phi}}=\int p(\mathbf{\Phi} / \mathbf{Y}) d \mathbf{\Phi} \\
p(\boldsymbol{\lambda} / \mathbf{Y})=p(\boldsymbol{\lambda}, \hat{\mathbf{\Phi}} / \mathbf{Y})
\end{array}\right.
$$

\section{Synthesis of the optimal spatial-temporal processing algorithm}

The expression of the optimal adaptive algorithm in the conditions of an unknown interference situation in the form (9) allows us to divide it into two stages:

- adaptation step (estimates a correlation matrix), 
- an evaluation phase informative parameters (orientation parameter).

Of course, the accuracy of algorithm (9) will depend on the accuracy of the estimation of the correlation matrix of interference $\hat{\mathbf{\Phi}}$.

Using the observation equation (7), we obtain the optimal algorithm for measuring the parameters $\boldsymbol{\lambda}$ of the signal $S_{c k}(\boldsymbol{\lambda})$ based on multi-channel observation $\mathbf{Y}$ in the time interval $t=\overline{1, T}$. The value of the interval $T$ does not have much significance, but we can set it equal to $1 \mathrm{~ms}-$ the main interval of GLONASS and GPS.

We restrict ourselves to considering the maximum likelihood of an $\hat{\lambda}$ a priori unknown parameter vector $\boldsymbol{\lambda}$ of the selected distribution, which is a solution to the system of likelihood equations.

The conditional probability density necessary for calculating the likelihood ratio $p\left(\mathbf{Y} / \mathbf{S}_{c}(\boldsymbol{\lambda})\right)$ can be obtained from the known multidimensional distribution density $[1-6] p(\dot{\mathbf{Y}})$. If there is a signal in sample $\mathbf{Y}$, characterized by a nonzero mathematical expectation:

$$
M[\mathbf{Y}]=M\left[\mathbf{S}_{c}(\lambda)\right]+M[\mathbf{n}(v)]=M\left[\mathbf{S}_{c}(\lambda)\right]=\mathbf{S}_{c}(\lambda) .
$$

In this case, each discrete $y_{k}$ of the sample $\mathbf{Y}$ receives an increment $S_{c i}(\lambda)$, and the distribution $p_{\text {сп }}\left(\dot{\mathbf{Y}}^{*}\right)$ is shifted by the value of the mathematical expectation $\mathbf{S}_{c}(\lambda)$. As a result

$$
p_{\text {cп }}(\dot{\mathbf{Y}})=p_{n}\left\{\left[\dot{\mathbf{Y}}-\mathbf{H} \dot{\mathbf{S}}_{c}(\boldsymbol{\lambda})\right]\right\} .
$$

Composing the logarithm of the likelihood ratio $\ln l(\lambda)=\ln \left[p_{\mathrm{c}}(\dot{\mathbf{Y}}) / p_{\text {сп }}(\dot{\mathbf{Y}})\right.$ and substituting equation (11) into it, we obtain

$$
\begin{gathered}
\left.\ln l(\boldsymbol{\lambda})=-\left[\dot{\mathbf{Y}}-\mathbf{H} \dot{\mathbf{S}}_{c}(\boldsymbol{\lambda})\right)\right]^{* T} \dot{\boldsymbol{\Phi}}^{-1}\left[\dot{\mathbf{Y}}-\mathbf{H} \dot{\mathbf{S}}_{c}(\boldsymbol{\lambda})\right] / 2+\dot{\mathbf{Y}}^{* T} \dot{\boldsymbol{\Phi}}^{-1} \dot{\mathbf{Y}} / 2= \\
=\dot{\mathbf{Y}}^{* T} \dot{\boldsymbol{\Phi}}^{-1}\left[\mathbf{H} \dot{\mathbf{S}}_{c}(\boldsymbol{\lambda})\right] / 2+\left[\mathbf{H} \dot{\mathbf{S}}_{c}(\boldsymbol{\lambda})\right]^{* T} \dot{\boldsymbol{\Phi}}^{-1} \dot{\mathbf{Y}} / 2-\left[\mathbf{H} \dot{\mathbf{S}}_{c}(\boldsymbol{\lambda})\right]^{* T} \dot{\boldsymbol{\Phi}}^{-1}\left[\mathbf{H} \dot{\mathbf{S}}_{c}(\boldsymbol{\lambda})\right] / 2 .
\end{gathered}
$$

In this equation the first two terms form the sum of complex conjugate quantities. After transposition and complex conjugation of the first term, the latter is reduced to type $\left[\mathbf{H} \dot{\mathbf{S}}_{c}(\boldsymbol{\lambda})\right]^{* T} \dot{\boldsymbol{\Phi}}^{-1} \dot{\mathbf{Y}} / 2$. In this case, the Hermitian property of the inverse matrix was taken into account $\left(\dot{\boldsymbol{\Phi}}^{-1}\right)^{* T}=\dot{\boldsymbol{\Phi}}^{-1}$. As a result, the sum of the first two terms (11) will be equal to

$$
\zeta=\operatorname{Re} \dot{\mathbf{Z}}(\boldsymbol{\lambda})
$$

where

$$
\dot{\mathbf{Z}}(\boldsymbol{\lambda})=\dot{\mathbf{Z}}=\left[\mathbf{H} \dot{\mathbf{S}}_{c}(\boldsymbol{\lambda})\right]^{* T} \dot{\boldsymbol{\Phi}}^{-1} \dot{\mathbf{Y}}=\dot{\mathbf{Y}}^{T}\left(\dot{\boldsymbol{\Phi}}^{-1}\right)^{*}\left[\mathbf{H} \dot{\mathbf{S}}_{c}(\boldsymbol{\lambda})\right]^{*}
$$

Introducing the notation

$$
q^{2}(\boldsymbol{\lambda})=\frac{\left[\mathbf{H} \dot{\mathbf{S}}_{c}(\boldsymbol{\lambda})\right]^{* T} \dot{\boldsymbol{\Phi}}^{-1}\left[\mathbf{H} \dot{\mathbf{S}}_{c}(\boldsymbol{\lambda})\right]}{2} .
$$

Relation (12) can finally be written in the form

$$
\ln l(\boldsymbol{\lambda})=\zeta(\lambda)-q^{2}(\lambda) / 2 .
$$

The parameter $q^{2}(\lambda)$ in (16) is purely real and does not depend directly on the received signals. It is proportional to the energy of the useful signal at the input of the processing device 
The first term in equation (16) represents the real part of the complex weighted sum, which depends on the discrete of the received signal $\mathbf{Y}$.

$$
\dot{\mathbf{Z}}(\boldsymbol{\lambda})=\sum_{k=1}^{T} \dot{\mathbf{Y}}_{k}^{* T}\left(\dot{\boldsymbol{\Phi}}_{k}^{-1}\right)\left[\mathbf{H} \dot{\mathbf{S}}_{c k}(\boldsymbol{\lambda})\right]
$$

It follows from the foregoing that the likelihood ratio and its logarithm are primarily determined by the weighted sum $\zeta=\operatorname{Re} \dot{\mathbf{Z}}(\boldsymbol{\lambda})$, the calculation of which determines the first and main stage of processing the received oscillation $\mathbf{Y}$. The weighted sum $\zeta(\lambda)$ determines those essential operations that are necessary to extract from the received implementation $\mathbf{Y}$ all information about the parameters of the received signal. Therefore, $\zeta(\lambda)$ together with $\ln l(\lambda)$ is also sufficient statistics.

Given the independence of noise in the received signals (7) at different points in time, the observation functional of the entire set of observations $\mathbf{Y}_{k}^{T}$ in the interval is [1]

$$
\dot{\mathbf{Z}}(\boldsymbol{\lambda})=\sum_{k=1}^{T} \dot{\mathbf{Y}}_{k}^{* T}\left(\dot{\phi}_{k}^{-1}\right)\left[\mathbf{H} \dot{\mathbf{S}}_{c k}(\boldsymbol{\lambda})\right]
$$

The evaluation $\hat{\lambda}=\max _{\lambda}{ }^{-1} p\left(\mathbf{Y}_{k}^{T} \mid \boldsymbol{\lambda}\right)$ will be determined

$$
\begin{aligned}
\hat{\boldsymbol{\lambda}}=\max _{\lambda}{ }^{-1} \operatorname{Re}\{\operatorname{lnl} l(\boldsymbol{\lambda})\} & =\max _{\lambda}{ }^{-1} \operatorname{Re}\{\zeta(\boldsymbol{\lambda})\}=\max _{\lambda}{ }^{-1} \operatorname{Re}\left[\mathbf{H} \dot{\mathbf{S}}_{c}(\boldsymbol{\lambda})\right]^{* T} \dot{\boldsymbol{\Phi}}^{-1} \dot{\mathbf{Y}}= \\
= & \max _{\lambda}{ }^{-1} \operatorname{Re}\left\{\mathbf{H}^{*} \boldsymbol{\Phi}^{-1} \mathbf{Y}_{k} \mathbf{S}_{k}^{* T}(\boldsymbol{\lambda})\right\} .
\end{aligned}
$$

Denoting

$$
\mathbf{H}_{k}=\mathbf{H}^{*} \boldsymbol{\Phi}^{-1} \mathbf{Y}_{k},
$$

we find that the estimate $\hat{\boldsymbol{\lambda}}$ corresponds to the maximum estimate of the correlation integral [1]

$$
\hat{\boldsymbol{\lambda}}=\max _{\lambda}{ }^{-1} \operatorname{Re}\left\{\sum_{k=1}^{T} \boldsymbol{\eta}_{k} \mathbf{S}_{c k}(\boldsymbol{\lambda})\right\}
$$

which is the usual signal processing algorithm in the time domain.

Observations from the outputs of the elements of the antenna $\mathbf{Y}_{k}$ are included in algorithm (21) only combined into the equivalent observation $\boldsymbol{\eta}_{k}$. Equation (21) represents the optimal spatial processing algorithm in a multi-element antenna array. Denoting $\mathbf{W}=\boldsymbol{\Phi}^{-1} \mathbf{H}$, we write the equivalent observation in the form of a weighted sum of the outputs of the elements of the antenna array

$$
\boldsymbol{\eta}_{k}=\mathbf{W}_{k}^{* \mathrm{~T}} \mathbf{Y}_{k}=\sum_{l=1}^{m} w_{l k}^{*} y_{l k},
$$

weighting factors in the sum (22) are complex numbers. The estimate $\hat{\boldsymbol{\lambda}}$ does not change when $\mathbf{W}$ is multiplied by an arbitrary positive (non-complex) number. Sometimes it is convenient to consider $\mathbf{W}$ in the form $[1,2]$

$$
\begin{gathered}
\mathbf{W}=D \boldsymbol{\Phi}^{-1} \mathbf{H}, \\
D=1 / \mathbf{H}^{*} \boldsymbol{\Phi}^{-1} \mathbf{H} .
\end{gathered}
$$

Since in this case $\mathbf{W H}=\mathbf{H}^{*} \boldsymbol{\Phi}^{-1} \mathbf{H} / \mathbf{H}^{*} \boldsymbol{\Phi}^{-1} \mathbf{H}=1$, then

$$
\boldsymbol{\eta}_{k}=\mathbf{W}^{*} \mathbf{Y}_{k}=\dot{\mathbf{S}}_{c k}(\boldsymbol{\lambda})+\mathbf{n}_{e q},
$$


where $\mathbf{n}_{e q}$ is the equivalent noise with dispersion $D \eta=M\left\{\mathbf{n}_{e q} \mathbf{n}_{e}^{*} q\right\}=M\left\{\mathbf{W}^{*} \mathbf{n}_{e q} \mathbf{n}_{e}^{*} q \mathbf{W}\right\}=$ $=\mathbf{W}^{*} \boldsymbol{\Phi} \mathbf{W}=\mathbf{H}^{*} \boldsymbol{\Phi}^{-1} \boldsymbol{\Phi} \boldsymbol{\Phi}^{-1} \mathbf{H} / \mathbf{H}^{*} \boldsymbol{\Phi}^{-1} \mathbf{H}=1 / \mathbf{H}^{*} \boldsymbol{\Phi}^{-1} \mathbf{H}$, which coincides with equation (24).

It is important that the characteristics of the optimal parameter estimation $\hat{\lambda}$ in multichannel observation are equivalent to the characteristics of estimation $\hat{\lambda}$ in single-channel observation. The resulting optimal spatial-temporal processing algorithm is shown in Fig. 2 as a series-connected optimal spatial filter and a temporal filter [3].

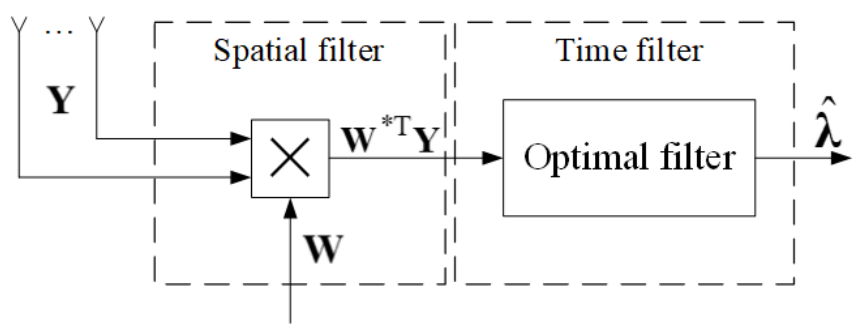

Fig. 2. Geometric interpretation of the location of the elements of the antenna array and received signals

\section{Conclusion}

In more general formulations of the problem, including signal parameters changing in time and parameters $\mathbf{H}_{t}$ and $\boldsymbol{\Phi}_{t}$ characterizing the spatial arrangement of the antenna array, navigation satellites and interference sources, only the form of the time filter changes in the structure of the optimal algorithm.

Thus, an algorithm for measuring navigation parameters in an unknown interference environment is obtained. The algorithm is divided into two stages: the adaptation stage-calculating the correlation matrix of interference based on spatial filtering algorithms and the stage of measuring navigation parameters based on time algorithms.

This work was supported by the Ministry of Science and Higher Education of the Russian Federation in the framework of the Federal target program "Research and development on priority directions of development of the scientific-technological complex of Russia for 2014-2020" (agreement no. 05.608.21.0274, unique ID project RFMEFI60819X0274).

\section{References}

[1] A.I.Perov, V.N.Harisov, GLONASS. The principles of construction and operation, Moscow, Radio Engineering, 2010 (in Russian).

[2] A.I.Perov, Methods and algorithms for the optimal reception of signals in the equipment of consumers of satellite radio navigation systems, Moscow, Radio Engineering, 2012 (in Russian).

[3] A.I.Perov, Statistical theory of radio systems, Moscow, Radio Engineering, 2003 (in Russian).

[4] V.N.Tyapkin, Determination of the angular orientation of an object from the radio navigation signals of spacecraft under the influence of intentional broadband interference, Successes of modern radio electronics. Foreign Radio Electronics, 9(2012), 100-106 (in Russian). 
[5] D.D.Dmitriev, V.N.Tyapkin, N.S.Kremez, Methods of adaptation of phased array antennas to interference in satellite radio navigation systems, Radio Engineering, 9(2013), 39-43 (in Russian).

[6] V.N.Tyapkin, D.D.Dmitriev, E.N.Garin, A.V.Sokolovsky, Operation of the amplitude-phase distribution of the adaptive phased array antenna, Industrial Automated Control Systems and Controllers, 4(2013), 66-71 (in Russian).

[7] Yu.L.Fateev, E.N.Garin, D.D.Dmitriev, V.N.Tyapkin, The use of filtering when measuring the angular orientation of a rotating object, Radio Engineering, 6(2013), 46-49 (in Russian).

[8] Ya.D.Shirman, Radio-electronic systems: Fundamentals of construction and theory. Directory. Ed. 2nd, rev. and add., Moscow, Radio Engineering, 2007 (in Russian).

[9] Ya.D.Shirman and [other], The first domestic studies of the adaptation of antenna systems to interfering influences, Radio engineering, 11(1989), 1989 (in Russian).

[10] V.N.Tyapkin, D.D.Dmitriev, V.G.Konnov, A.N.Fomin, A method for determining the vector of spectral coefficients by the likelihood ratio criterion, Bulletin of the Siberian state Aerospace University named after Acad. M.F. Reshetneva, 43(2012), no. 3, 76-79 (in Russian).

[11] V.I.Dzhigan, Equivalence conditions for recursive adaptive filtering algorithms by the least squares criterion, Telecommunications, 6(2006), 6-11 (in Russian).

[12] I.A.Lubkin, V.N.Tyapkin, The use of recurrent adaptive algorithms to solve the problem of suppressing active noise interference in satellite communication systems, Bulletin of the Siberian state Aerospace University named after Acad. M. F. Reshetneva, 28(2010), no. 2, 39-43 (in Russian).

\title{
Синтез измерителей параметров радионавигационных сигналов, принимаемых на разнесенные в пространстве антенные элементы в условиях помех
}

\author{
Валерий Н. Тяпкин \\ Дмитрий Д. Дмитриев \\ Николай С. Кремез \\ Игорь В. Тяпкин
}

Сибирский федеральный университет Свободный, 79, Красноярск, 660041

Россия

\begin{abstract}
Современная навигационная аппаратура ГНСС имеет существенный недостаток - низкую помехоустойчивость. Решение этой проблемы основано на применении антенн с базированной решеткой, использующих алгоритмы адаптивной обработки сигналов. Конечный эффект такой обработки определяется уровнем и полнотой исполъзования существующих различий, а такље качеством учета каждого из них в зависимости от степени известности статистических характеристик сигналов и помех. В статъе рассматривается теория синтеза алгоритма измерения параметров радионавигаџионных сигналов, принимаемых на разнесенные антенны при воздействии помех.
\end{abstract}

Ключевые слова: Фазированная антенная решетка, адаптивные алгоритмы, закон нормального распределения, вектор оцениваемых параметров, вектор полезных сигналов. 